J. Kestin

DOE/ER/13687--10

Brown University

Providence, RI USA

\title{
Internal Variables in the Local-Equilibrium Approximation
}

\section{Abstract}

The aim of this contribution is to explore the basis and consequences of the formalism known in the literature as the method of Inca! equilibrium. The view is taker that contemporary controversies regarding the foundations of thermodynamics are rooted nat only in different sets of concepts and principles, but also in semantics. Therefore, an aitempt is made here to use a consistent group of terms, each of whose dictionary meaning corresponds to its physical nature as closely as possible. Since the intention is to study irreversible processes in system in which even locally there prevails a state of nonequilibrium, the term local equilibrium is abandoned in favor of the phrase principle of local state.

In defining the therrnodynamic state of a system, a distinction is made between the intensive parameters which appear in the physical space (Kontaktgroessen) and those which describe states of constrained equilibrium in the Gibbsian phase (state) space. The latter consists of a set of extensive variables (internal energy $U$, external deformation parameters and the internal deformation variables $\alpha$ ) because, in contrast with intensive variables, they can be measured in equilibrium as well as in nonequilibrium. The properties and uses of the internal variables are outlined following P. W. Bridgman's proposal. 
The principle of local state is applied by associating with every nonequilibrium state $n$ an accompanying equilibrium state $e$ of equal values of $U, a, \alpha$, and by asserting that the entropy $\overline{\mathrm{S}}$ assignable in physical space and temperature $\bar{T}$ measured in it can be approximated by the values $S$ and $T$ calculated in the Gibbsian phase space by standard, classical methods.

A continuous sequence of accompanying equilibrium state: (curve $R$ in phase space) is called an accompanying reversible process, it is conceived as an adiabatic projection of the continuous sequence of nonequilibrium states which constitute the irreversible process $I$. This allows us to cast the classical Gibbs equation in rate form and to derive explicit expressions for the rate of entropy production $\dot{\theta}$ by eliminating the rate $d u / d t$ between it and the energy balance equation.

The nature of the approximation involved in this procedure is made explicit. Hence, the preference to speak of the local state approximation.

The essential part of the method consists in the formulation of the Gibbs equation for the accompanying reversible process in the phase space. This is obtained from the knowledge of the physics of the situation and leads to the identification of the internal deformation variables and the hypothetical virtual (i.e., reversible) work done against them. The Gibbs equation forms the basis for the derivation of an explicit form of the local rate of entropy production and a rational formulation of the rate equations between the generalized forces and fluxes which appear in it. The union between the rate equations and the fundamental equation relating the extensive variables of the constrained equilibrium states in phase space yields the constitutive law for the system. The constitutive law with the rate equations inserted into the appropriate conservation laws produces the set of partial differential equations which govern the process. Their solution, in the form of a set of time-dependent fields, subject to the appropriate boundary conditions constitutes the irreversible process under study. 
It is noted that the local-state approrimation, made explicit in this paper, has been used and tested in fluid mechanics though its validity is contested in contemporary continuum mechanics and mechanics of solids. The author takes the view that th se principal theories of irreversible processes, as they occur in engineering applications, fit into the same formalism, as expounded in the text. 


\section{Table of Contents}

Abstract i

1. Introduction 2

2. Semantics and Terminology 3

2.1. General 3

2.2. Equilibrium 3

3. Thermodynamic State 6

3.1. Physical Space 6

3.2. Internal Deformation Variables 7

3.3. The Concept of State in Equilibrium;
State Space

3.4. The Concept of State in Nonequilibrium 13

4. Principle of Local State 14

4.1. Accompanying Equilibrium State $\quad 14$

4.2. Geometric Illustration 15

4.3. Entropy Production 17

4.4. Rate Equations 19

4.5. A Digression 21

5. Nature of the Approximation 22

Captions to Figures 24

Figure 1. 25

Figure 2. 26

Figure $3 . \quad 27$

Figure 4. 28

Figure 5.

Appendix 1. 30

Appendix 2. 


\section{Introduction}

This symposium is witness to the fact that there exists no consensus among the practitioners of thermodynamics concerning the very basis of their subject. This, in spite of over two hundred years of evolution and in contrast with, for example, Newtonian mechanics or the younger disciplines, such as electromagnetic theory, chemical kinetics and other classical branches of physics.

The controversy is about the way to create a consistent description of irreversible processes and the treatment of nonequilibrium states closely connected with them. The key is a satisfactory formulation of the Second Law and of a measurable entropy of a state which is not in equilibrium.

A familiarity with the literature of the subject would suggest that research on the properties of fluids as well as fluid mechanics, including gas dynamics, seems to operate on one set of concepts and principles; these are often disputed by research workers whose background is in continuum and solid mechanics. Uncoubtedly, other "schools" will also be represented at the symposium. Needless to say, we do not believe in the objective existence of different, of ten conflicting, thermodynamics.

The purpose of this paper is to explore the formulation and consequences, in more detail as has yet been done, of the formulation which makes use of the concept of local equilibrium. This concept appeared quite natural, though only by implication, to, for exampie, J. Hadamard and P. Duhem in the nineteenth century. In the forties of this century, the resulting methodology was formalized in more detail by J. Meixner in his Handbuch article and by 1 . Prigogine in his doctoral thesis. Probably the most authoritative treatment can be found in the treatise Non-Equilibrium Thermodyramics by S. R. de Groot and P. Mazur. We shall defend the view that the phrase local equilibrium is grossly misleading, because we wish to summarize the relevant practices and

\footnotetext{
See here Appendix 1.
} 
equations as they apply to systems in which even locally the state departs from equilibrium by a larger or lesser distance in the presence of gradients of intensive properties, such as temperature, and whose extensive properties vary with time at higher or lower rates. When this is the case, the evolution in time constitutes an irreversible process. For these reasons, we shall prefer to speak of the application of the principle of local state. We shall concentrate on the intrinsic irreversibility of processes and describe it in terms of rigorously defined internal variables.

We shall defend the view that there is no difference between the thermodynamic principles which govern irreversible processes in fluids or solids.

\section{Semantics and terminology}

\subsection{General}

The controversies which have developed between the different "schools" are mainly due to the adoption of different concepts, but some of the difficulties can be traced to semantics. It is a fact that different authors use the same terms for different concepts as well as different names for identical concepts. It would not be practical to set up a clarifying glossary. We shall use an internally consistent set of concepts and terms making sure that a term's dictionary meaning corresponds to its physical nature as closely as possible. This was the case earlier when we preferreci to speak about the principle of local state rather than about the method of local equilibrium.

We must, however, make an exception for the concept of thermodynamic equilibrium as used in this paper.

\subsection{Equilibrium}

We shali say that a system (or any of its subsystems) is in thermodynamic equilibrium when it does not interact with its surroundings. For the sake of being definite, we shall limit attention to interactions through the transfer of work $W$ and 
heat $Q$ (so-called Schottky systems in W. Muschik's terminology). Under such conditions, its intensive and extensive properties are constant in time. It follows that the system does not sustain gradients of its intensive properties and that its extensive properties do not change with time. It is sometimes useful to realize that replacing (in imagination) the boundary of a system by an adiabatic wall or the equally imaginary insertion of any number of rigid adiabatic partitions into it changes nothing.

The state of thermodynamic equilibrium is maintained by a set of constraints whose specification forms an essential part of the specification of the system. It is assumed that any system confined within a rigid adiabatic boundary reaches thermodynamic equilibrium eventually.

This is the ideal picture. In the analysis of physical systems, we often encounter situations in which the constraints assume a more subtle form than material walls or partitions. For example, in a system which contains two reacting gases, say hydrogen and oxygen, the rate at which the chemical transformation occurs may become very slow leading to insignificant changes over periods of time $\tau$ which are long compared with the time of observation $\mathcal{J}$. We may then analyze the system as if it were in thermodynamic equilibrium. This can be formalized by introducing the relaxation time

$$
\tau=\left(\xi-\xi_{0}\right) / \dot{\xi}
$$

where $\xi$ is the extent of the reaction and $\dot{\xi}$ its rate.

If

$$
\tau \gg \mathcal{J},
$$

we say that the system is in thermodynamic equilibrium for all intents and purposes. Such would be the case for the $\mathrm{O}_{2}-\mathrm{H}_{2}$ mixture at a sufficiently low temperature. The rate of a process, here the reaction rate $\dot{\xi}_{j}$ can be affected by manipulating the constraints. In the case of a chemical reaction, we may think of slowing down the 
reaction by adding an anticatalyst to the system or speeding it up by adding a catalyst. Thus, we consider two extreme cases:

(a) in the presence of an anticatalyst, when $\tau \rightarrow \infty$ and condition (1a) is satisfied, we shall speak about a constrained state of thermodynamic equillibrium. This is often called frozen equilibrium.

(b) In the presence of a catalyst, when $\tau \rightarrow 0$ we would have

$$
\tau \ll \mathcal{I} \text {. }
$$

In this case the reaction is said to reach an unconstrained state of inermodynamic equilibrium. Semantic problems occur, because some authors would refer to case (1b) as equilibrium tout court and may never have to deal with frozen equilibrium.

The case of metastable equilibrium also leads to semantic misunderstandings. Metastable equilibrium differs from stable equilibrium (constrained or unconstrained) merely by the way its constraints are manipulated. Normally, as in the examples above, the operator has full control over the constraints. Metastable equilibrium does not differ from stable equilibrium while it lasts. However, its internal constraints may remove themselves spontaneously, so that subsequently the system reaches another state, this time one of stable equilibrium.

We take the view that the preceding distinctions apply generally and not only to chemical reactions or fast expanding fluids.

It is tedious always to have to specify the form of equilibrium under discussion, and most authors are satisfied with the single term: equilibrium. We shall follow this custom, but will use the more detailed terms if the potential for a misunderstanding is suspected.

\section{DISCLAIMER}

\footnotetext{
This report was prepared as an account of work sponsored by an agency of the United States Government. Neither the United States Government nor any agency thereof, nor any of their Government. Neither the United express or implied, or assumes any legal liability or responsiemployees, makes any warranty, express or implied, or assumes any legal liability or respons, bility for the accuracy, completeness, or usefulness of any information, apparad rights. Referprocess disclosed, or represents that its use would not infringe privately owed hights. Refer ence herein to any specific commercial product, process, or service by trade name, traderement, recommanufacturer, or otherwise does not necessarily constitute or imply its endorsemen, The views mendation, or favoring by the United States Government or any agency thereof. The views and opinions of authors expressed herein do not United States Government or any agency thereof.
} 


\section{Thermodynanic state}

\subsection{Physical space}

Attention is centered on a continuum $B$, contained within a boundary $\partial Q$, Figure 1, and on an element ("point") $\Delta V$ in it. We shall describe its state by indicating its internal energy $U$, a set of defor mation parameters $a(x, t)$, which determine the work transfer."

$$
d W=\bar{F} \cdot d a \quad \text { (or } \dot{W}=\bar{F}_{i} \dot{a}_{i} \quad \text { summation intended) } .
$$

Here $\bar{F}(x, t)$ is a set of conjugate generalized forces, intensive in nature, as measured in the physical space of Figure 1. The heat interaction is described by a field of heatflux vectors $q(x, t)$ associated with a temperature field $\bar{T}(x, t)$.

In fluids, $\dot{a}$ is represented by the symmetric rate-of-deformation tensor $\rho^{-1} \dot{\varepsilon}_{i j}(x, t)$ and $\bar{F}$ is its conjugate symmetric Cauchy stress tensor $\bar{\sigma}_{i j}(x, t)$. In solid continua it is the deformation tensor $\varepsilon_{\mathrm{ij}}$ itself.

It should be emphasized that $\bar{F}, \bar{T}$ and $\bar{\sigma}$ are thought of here as contact quantities (Kontaktgroessen) in the sense of W. Muschik.

Since the element $\Delta V$ interacts with its surroundings, it is not in equilibrium and its evolution in time constitutes an irreversible process. We shall think of irreversible processes as being continuous sequences of nonequilibrium states in time.

The parameters mentioned above enter into the equation of energy conservation (First Law of thermodynamics) which we write:

$$
\text { for fluids } \quad \rho \frac{d u}{d t}=\bar{\sigma}_{i j} \frac{\partial v_{i}}{\partial x_{j}}-\frac{\partial q_{k}}{\partial x_{k}} \text {, }
$$

\footnotetext{
Work done by the system on the surroundings will be reckoned positive. In solid mechanics it is more common to follow the opposite convention.
} 
for solids $\rho \frac{d u}{d t}=\bar{\sigma}_{i j} \frac{d \varepsilon_{i j}}{d t} \cdot \frac{\partial q_{k}}{\partial x_{k}}$.

Here, $v(x, t)$ is the velocity field in the fluid, $p$ is the density, and $u$ the internal energy per unit mass.'

In the more primitive notation employed in elementary textbooks, these equations are writter.

or

$$
\begin{aligned}
& d U=d Q \cdot d W \\
& d U=d Q \cdot \bar{F} \cdot d a .
\end{aligned}
$$

The variables introduced so far are inadequate to formulate a closed system of partial differential equations whose solutions as functions of time would describe the irreversible process under discussion. To be in a position to do so, we introduce a set of internal deformation variables $\alpha$, which characterize the dissipative mechanisms operating inside the continuum as it is deformed by the generalized external forces $\bar{F}$. We have consistently advocated the use of one of Bridgman's insights to clarify the role of these parameters.

\subsection{Internal deformation variables}

The reality of internal variables has been, and still is, disputed. The opposition is partly due to the unfortunate term: hidden variables, used by some early authors.

Observation suggests that each system which performs an irreversible process due to internal dissipation is the seat of potential deformations $\alpha_{i}$. Under the action of forces $F$, external to it, the internal sites deform at some rate $\dot{\alpha}_{i}$, the movement along it occurring in the absence of balancing forces; the movement is "unresisted." The neighborhood of such a site shows locally increased temperatures resulting in internal heat fluxes. The element $\Delta V$ may develop local velocity fields or vibrations which often result in the production of sound waves ("pings" in plastically deformed metallic samples), etc., etc. 
Examples of such mechanisms occur in plastic deformation, in the creation of cracks, or in zones of shear in a flow. The movement of tectonic plates during an earthquake affords another example. The sketch in Figure 2 gives (a pale) idea of such a site. P. W. Bridgman's suggestion was to imagine a Gedankenexperiment which would allow us to think that the deformations $d \alpha_{j}$ have been balanced. This we imagine done by the introduction from the outside of fictitious conjugate forces $A_{j}^{\circ}$, which locally balance the system and impose equilibrium. It is clear that during such an experiment, the external conjugate generalized forces $\overline{F_{i}}$ change their magnitudes, say to $F_{i}^{\circ}$.

In a system thus equilibrated the external forces $F_{i}^{\circ}$ acting on $a_{i}$ and the fictitious, externally connected, forces $A_{j}^{0}$ acting on $\alpha_{j}$ can perform virtual work

$$
d W_{1}^{\circ}=F_{i}^{\circ} d a_{i} \quad \text { and } \quad d W_{2}^{\circ}=A_{j}^{0} d \alpha_{j} . \quad \text { (sums) }
$$

In elementary thermodynamics the phrase is used that the elements of virtual work $\mathrm{dW}^{\circ}$ have been performed reversibly. Clearly, there arises here a logical sleight of hand, puzzling to beginning students," which suggests that a system, now in equilibriur., performs a process nevertheless. The beginner's uneasiness is assuaged by the pretense that the steps $d a$ and $d \alpha$ have been imposed very slowly or, as the saying goes, quasistatically, meaning that asymptotically $\dot{a} \rightarrow 0$ and $\dot{\alpha} \rightarrow 0$.

In our presentation we shall avoid such misleading semantics and merely treat the term reversible work as a synonym for virtual work. For our purposes it is sufficient to know that under the action of the forces $F^{\circ}$ and $A^{\circ}$ the system is in equilibrium and that the virtual (now called reversible) work $(5 a, b)$ is done by the system on the surroundings when positive. Disputing ingrained habits in some expositions of the subject, we emphasize that the forces $A_{j}^{0}$ do not exist in the physical space (for this reason they failed to appear in Figure 1), and are fictitious.

\footnotetext{
The sophomore's dilemma.
} 


\subsection{The concept of state in equilibrium; state space}

We now consider the element $\Delta \mathrm{V}$ brought into a state of constrained equilibrium in Section 3.2. The constraints consist of the external forces $F^{\circ}$ and the fictitious forces $A^{\circ}$

Experience suggests that the state is specified uniquely by the indication of a set of extensive properties, namely its internal energy $U$, its external deformations $a$, and the internal parameters $\alpha$. We can now wrile the equivalent of Equations $(4 a, b)$ in the form.

$$
\begin{aligned}
d U & =d Q^{\circ}-d W^{\circ} \\
& =d Q^{\circ}-F_{i}^{\circ} d a_{i}-A_{j}^{\circ} d \alpha_{j} .
\end{aligned}
$$

Here, as before and later, we imply the summation convention for repeated indices. The essence of Part One of the Second Law of thermodynamics is to assert that the Pfaffian form

$$
d Q^{\circ}=d U+F_{i}^{0} d a_{i}+A_{j}^{0} d c_{j}
$$

possesses an integrating denominator $T(U, a, \alpha)$, which converts it to the perfect differential

$$
\mathrm{dS}(U, \mathbf{a}, \alpha)=\frac{\mathrm{d} U+F_{i}^{\circ} d a_{i}+A_{j}^{0} d \alpha_{j}}{T(U, a, \alpha)}
$$

of the function

$$
S=S(U, a, \alpha)
$$

called entropy. For this audience it is hardly necessary to repeat that of the infinity of integrating denominators we usually select one which is congruent to the empirical

* The fictitious generalized forces $\mathrm{A}^{\circ}$ appear in different guises, sarh under a different name, in the various branches of thermodynamics. They are called affinities in chemical thermodynamics and energy release rates in damage mechanics (also the force acting on a crack). In some branches of solid mechanics, they are known as Eshelby or configurational forces. In many treatises on irreversible processes, the term affinity is favored. We shall do likewise. 
temperature, and that $T$ in Equation (6d) is not necessarily the same as $\bar{T}$ measured in the physical space. Similarly, we recall that the function $S(U, a, \alpha)$ is invertible to

$$
U=U(S, a, \infty)
$$

and that the Gibbs equation

$$
d U=T d S-F_{i}^{0} d a_{i} \cdot A_{j}^{0} d a_{j}
$$

is fully equivalent to (6d).

It is very convenient to define a state space, the Gibbsian phase space, whose cordinates are the extensive properties

$$
U, a, \alpha \text {. }
$$

This is a manifold of $2+m$ dimensions in fluid mechanics and of $7+m$ dimensions in solid mechanics ( $\varepsilon_{i j}$ is symmetric!)." Here $m$ denotes the number of internal deformation variables in the description of the system. It is proved that in it the surfaces $S(U, a, \alpha)$ must be convex for intrinsic stability.

We re-emphasize that every point in the relevant domain of the phase space is a state of constrained equilibrium and that a continuous line $C$ which may be introduced into it to calculate line integrals is a sequence of equilibrium states. Later we shall provide an interpretation which will allow us to speak of such lines as reversible processes which are images (i.e., projections) of irreversible processes into the phase space. Evidently, the entropy of a system is calculated with the aid of the integral

$$
S(U, a, \alpha) \cdot S_{0}=\int_{C} \frac{d Q^{\circ}}{T}=\int_{C} \frac{d U+F_{i}^{0} d a_{i}+A_{j} d \alpha_{j}}{T},
$$

\footnotetext{
- Proponents of the less conventional versions of thermodynamics often make proposals for an enlargement of the manifold of states so that it would also include states of nonequilibrium. This would result in a state space of more than the $2+m$ or $7+m$ dimensions mentioned above. In this paper such an extension is emphatically exciuded. It is expected that the merits and consequences of adopting an enlarged state space will be discussed at this symposium.
} 
where $S_{0}$ is an arbitrary constant. This definition fully conforms with A. Sommerfeld's criterion quoted in Appendix 1.

At the risk of boring this audience, we recall that the intensive conjugate variables are uniquely determined by, say, the function ( $6 f)$, so that

$$
T(S, a, \alpha)=\frac{\partial U(S, a, \alpha)}{\partial S}, F_{i}^{0}=-\frac{\partial U(S, a, \alpha)}{\partial a_{i}}, A_{j}^{0}=-\frac{\partial U(S, a, \alpha)}{\partial \alpha_{j}}, \quad(9 a, b, c)
$$

tha! Maxwell's relations of the type

$$
\frac{\partial F_{i}^{\circ}}{\partial a_{j}}=\frac{\partial F_{i}^{\circ}}{\partial a_{i}} \text {, etc. }
$$

an be put to good use, and that Legendre transforms can be constructed. The useful ones are:

the Helmholtz free energy

$$
F=U \cdot T S \text { and } d F=-S d T-F_{i}^{0} d a_{i}-A_{j}^{0} d \alpha_{j}
$$

where

$$
F=F(T, a, \alpha)
$$

and the Gibbs free energy with $i=1$ (one work term $F^{\circ}$ da)

$$
G=U+F^{\circ} a-T S \text { and } d G=-S d T+a d F^{\circ}-A_{j}^{0} d \alpha_{j} \text {, }
$$

where

$$
G=G\left(T, F^{\circ}, \alpha\right)
$$

Evidently, for each of the potentials $F, G$ we may write relations analogous to $(9 a, b, c)$ and $(10)$. Although the affinities $A_{j}$ appear in each potential, the independent 
variables in them change as one potential is replaced by another. Explicit functions of the type of Equations (6e), (6f), (11c), (12c) are called fundamental equations because all equilibrium properties of systems and materials can be derived from any one of them by differentiation alone, i.e., without the need to introduce additional information.

It is worth noting for future reference that only coordinates of the Gibbsian phase space for internal energy, or what amounts to the same, for entropy, are extensive in character. The potentials $\mathrm{F}$ and $\mathrm{G}$ involve one or more intensive independent variable. Contemporary solid mechanics" is restricted, in most treatises, to isothermal processes with $\mathrm{dT}=0$, so that

$$
d F=-\rho^{-1} \sigma_{i j}^{o} d \varepsilon_{i j}-A_{j}^{0} d \alpha_{j}, \quad j=1,2 \ldots, m .
$$

The relevant Gibbsian phase space of six plus $m$ dimensions ( $\varepsilon_{i j}$ is symmetric!) is also composed of extensive independent variables.

Classically trained thermodynamicists are inclined to say that only functions defined in a Gibbsian phase space, particularly the entropy $S$ used so far, and their derivatives constitute (true) thermodynamic variables (or properties). Whether the reader subscribes to such a terminology or not, he will do well to bear their special significance constantly in mind. Thermodynamic tables and charts list exclusively such properties of equilibrium states.

The relevance of the preceding two sets of remarks will become clear in Section

\section{4 .}

- The dimension of $A^{\circ}$ in this equation is not the same as in previous equations where $\left[A^{\circ}\right]=N$. Here $\left[A^{\circ}\right]=N / k g$, if $[\alpha]=m$. In some problems different dimensions are more convenient. We shall disregard these subtleties in the remainder of this paper.

Whereas $\varepsilon_{i j}$ by itself cannot be said to be extensive, the group $\varepsilon_{i j} / \rho$ is extensive. 


\subsection{The concept of state in nonequilibrium}

We now resume our discussion of the physical space, Section 3.1, and observe that in order to be able consistently to apply the principles of thermodynamics to irreversible processes, it is necessary to answer two questions. First, how should we describe the state of the element $\Delta V$ in the absence of equilibrium in it, and especially of an entropy $\bar{S}$ that could be assigned to it, given that the definition in Equations $(6 c, d)$ is not applicable here Secondly, we must answer the question on how to formulate the equation of entropy balance.

To answer the first question, we shall postulate that if such a quantity exists, it can be approximated by the pplication of the principle of local state.

To answer the second question, we shall make further use of the principle of local state to derive an explicit equation for the rate of entropy production $\dot{\Theta}$, which then completes the so-called Clausius-Duhem inequality.

We note in Figure 1 that we can measure (in Sornmerfeld's sense, Appendix 1) the internal energy $U$ and the external deformation variables a, which are completely under the experimenter's control. We assert that we can also measure, in the same sense, the internal deformation variables $\alpha$, even though we cannot control them ${ }^{*}$ in the absence of the fictitious forces $\mathrm{A}^{\circ}$ in the physical space; they evolve spontaneously, but in an observable way. This is one more statement which many research workers dispute. The discovery of the number and nature of the $\alpha$ in any particular case belongs to the process of understanding of the physics of the problem under study. One way that leads to it is to invent the accompanying reversible process (defined in Section 4.1) through a Gedankenexperiment. We have achieved this goal for a number of systems studied in

The reader may wish to refresh his memory and re-read P. W. Bridgman's original suggestion, given here as Appendix 2. 
solid mechanics. One example is developed in a companion paper presented at this Symposium."

To complete the description of the state, if we may extend this term to nonequilibrium, it is necessary to specify its departure from equilibrium by the quantities which describe its interaction with the surroundings, for example, but not only, the heat flux vector q. Again, what additional quantities may be needed is a matter of understanding the physics. Whatever stand we take, it is evident that the equivalent of a fundamental equation cannot be formulated. For this reason, the intensive variables $\bar{F}$, $\bar{T}$, or its entropy $\bar{S}$, cannot be determined by analogs of the equations displayed in Section 3.3. Some thermodynamicists will search for an answer in what they call a constitutive law. We provide the answer through the principle of local state and the concepts of an accompanying equilibrium state e to a given nonequilibrium state $n$ and an accompanying reversible process $\mathrm{R}$ to a given irreversible process $\mathrm{I}$. These ideas will allnw us to forge a link to the Gibbsian state space and to the creation of a rational basis for the formulation of the constitutive law of which a fundamental equation of state is the first component.

\section{Principle of local state}

\subsection{Accompanying equilibrium state}

We recall that the set of extensive variables $U, a, \alpha$ is sufficient uniquely to specify a thermodynamic equilibrium state $e$ in phase space. We now center attention on a nonequilibrium state $n$ in the physical space which has identical values of the extensive variables, viz.

$$
U(n)=U(e), \quad a(n)=a(e), \quad \alpha(n)=\alpha(e) .
$$

\footnotetext{
Thermodynamic madelling of damage in elastic solids, by E. Honein, T. Honein, J. Kestin, and G. Herrmann.
} 
We can imagine that $e$ arises from $n$ by the Gedankenexperiment illustrated in Figure 4 . We stipulate that the element $\Delta V$ has been conrined to a rigid adiabatic enclosure which constrains the values of a. Simultaneously, we constrain the values of $\alpha$, say, in a chernically active system by an anticatalyst, or its equivalent, in other systems. The resulting state e of constrained (frozen) equilibrium will retain the value of internal energy $U$, because along the process $n \rightarrow e$ there is no exchange of heat or work ( $a=$ const, $\alpha=$ const). During such a process $\bar{T}$ and $\bar{F}$ will "relax" to $T$ and $F^{\circ}$, respectively, the temperature will become homogeneous, and $q$ will vanish. In this accompanying equilibrium state the internal deformation will be constrained by the forces $\mathrm{A}^{\circ}$ and virtual changes $\mathrm{dU}$, $\mathrm{da}$, $\mathrm{d} \alpha$ will satisfy the Gibbs Equation (6g), thus defining an entropy $S$ as in Equation (6e).

The essence of the principle of local state is to accept the approximation that the entropy $S$ and the temperature $T$ of state e can be used to characterize the nonequilibrium state $n$, or

$$
\bar{T}=T \text { and } \bar{S}=S .
$$

A perceptive reader will notice that the general principle of entropy increase demands that in Figure 4 we must have

$$
S(e)>\bar{S}(n) \quad \text { or } S(e)-\bar{S}(n)=\sigma \geq 0 \text {. }
$$

This is precisely why we speak of the local-state approximation. We revert to this point in Section 5.

\subsection{Geometric illustration}

It has been made clear that a nonequilibrium state $\mathrm{n}$ of a real irreversible process $I$ is not represented by the accompanying equilibrium state $e$, although the two share the same values of their extensive properties $U, a, \alpha$, Equation (14). The difference is in the conjugate intensive properties $T, F(A$ does not appear in $n$ ) and in their gradients as well 
as in the heat flux (which do not appear in e). In short, $n$ needs more variables for the description of its state than does e.

Figure 5 contains a geometric illustration of the relation of state $n$ to the space of equilibrium states $\mathrm{e}$. Since state $\mathrm{n}$ requires additional variables for its specification, the corresponding point requires a state space of higher dimensionality than does e whose space serves as a base for $n$. The base manifold $e(U, 2, \alpha)$ symbolizes the hyperspace of equilibrium states, and state $\mathrm{n}$ is placed on curve 1 . The latter represents a projection of an irreversible process of which $n$ is one intermediate state. The axis $z$ symbolizes the variables which are needed for the specification of $n$ beyond the set $U, a, \alpha$. During the hypothetical process $\mathrm{n} \rightarrow$ e discussed in the preceding Section (Figure 4), only states $z$ change ("relax") so that we regard $e$ as an adiabatic projection of $n$. The locus $R$ of all accompanying equilibrium states $e$ to the sequence of states on I represents the accompanying reversible process to $\mathrm{I}$. We re-emphasize that all states along $R$ are equilibrium states by the nature of the construction, and the question arises as to how one equilibrium state $e$ can be thought of as reaching to its neighbor when all are in equilibrium. This is the beginner's dilemma discussed at the end of Section 3.2. At this stage we postulate that the rate at which I progresses in time is impressed on $R$. We picture here a reversible process which occurs at a finite rate and not "infinitely slowly" or one that must be the result of convergence from a real process when its rate is reduced to zero. I do not think that the concept of a reversible process which occurs at a finite rate does violence to our physical imagination any more than does using Euclidean geometry to describe the spatial relations in the "real world." The question on how to approximate a real process to a condition of reversibility is analogous to the question of how to realize a Euclidean point, line, or plane in a physical structure. 


\subsection{Entropy production}

The discussion in the preceding two sections serves as a motivation for the conversion of the Gibbs equation (6g) to a rate form,

$$
\frac{d u}{d t}=T \frac{d s}{d t}-F_{i}^{\circ} \frac{d a_{i}}{d t}-A_{j}^{0} \frac{d a_{j}}{d t} .
$$

Introducing quantities per unit mass (lower-case symbols) and specializing, wie would write

(a) in fluid mechanics

$$
\frac{d u}{d t}=T \frac{d s}{d t} \cdot p \frac{d(1 / \rho)}{d t} \cdot A_{m}^{\circ} \frac{d \alpha_{m}}{d t}
$$

(b) in solid mechanics"

$$
\frac{d u}{d t}=T \frac{d s}{d t}+\frac{1}{\rho} \sigma_{i j}^{0} \frac{d \varepsilon_{j j}}{d t}-A_{m}^{0} \frac{d \alpha_{m}}{d t}
$$

Here $p$ is the equilibrium pressure in a fluid.

The balance of entropy is established by juxtaposing these equations on the expressions $(3 a, b)$ for the First Law, in that $d u / d t$ is eliminated between them. This can be done by virtue of the adoption of the principle of local state according to which the designated quantities appearing in $(17 \mathrm{a}, \mathrm{b})$ can be applied to the nonequilibrium states of $(3 a, b)$.

I think that there is no need to record the intermediate steps for this audience. As an additional step we accept the interpretation that the entropy flux $\eta$ is equal to $q / T$ and arrange the resulting terrns in the form of an entropy balance. In either case, we obtain

- Forbidden in many presentations of thermodynamics

In this equation, as is cust mary in solid mechanics, work done on the system is positive. 


$$
\rho \frac{d s}{d t}+\frac{\partial}{\partial x_{k}} \frac{q_{k}}{T}=\dot{\theta}, \dot{\theta} \geq 0
$$

where $\dot{\theta}$ is the local rate of entropy production. Equation (18) has the form which some authors describe as the Clausius-Duhem inequality. The Second Part of the Second law of thermodynamics mandates that the rate of entropy production must be positive as indicated; it may vanish in a hypothetical reversible process.

From the practical point of view, the most important result of the local-state approximation is that the establishment of the entropy balance, equation (18), yields explicit expressions for $\dot{\Theta}$. These are:

(a) in fluid mechanics

$$
\dot{\theta}=q_{k} \frac{\partial}{\partial x_{k}} \frac{1}{T}+\frac{\bar{\sigma}_{i j} \varepsilon_{i j}^{\prime}}{T}+\frac{P+\bar{\sigma}^{\prime \prime}}{T} \frac{\partial v_{i}}{\partial x_{i}}+\frac{\rho A_{m}^{\circ}}{T} \dot{\alpha}_{m} ;
$$

(b) in solid mechanics

$$
\dot{\theta}=q_{k} \frac{\partial}{\partial x_{k}} \frac{1}{T}+\frac{\bar{\sigma}_{i j}-\sigma_{i j}^{0}}{T} \varepsilon_{i j}+\frac{\rho A_{m}^{0}}{T} \dot{\alpha}_{m} .
$$

In equation (18a) we introduced the customary transformations

$$
\frac{\partial v_{i}}{\partial x_{j}}=\dot{\varepsilon}_{i j}+\dot{\omega}_{i j} ; \bar{\sigma}_{i j}=\bar{\sigma}_{i j}^{\prime}+\delta_{i j} \bar{\sigma}_{i}^{\prime \prime} \dot{\varepsilon}_{i j}=\dot{\varepsilon}_{i j}^{\prime}+\delta_{i j} \frac{\partial v_{i}}{\partial x_{i}} . \quad(19 a, b, c)
$$

Here $\dot{\varepsilon}_{i j}$ is the symmetric rate-of-strain tensor and the antisymmetric $\dot{\omega}_{i j}$ describes the, irrelevant here, rigid-body rotation. Primes denote deviators and $\sigma^{\prime \prime}$ is the trace of $\dot{\sigma}_{\mathrm{ij}}$. 


\subsection{Rate equations}

The consequences and inte. pretations, discussed below, which follow from Equations (18a, b) are commonly accepted in fluid mechanics. They are disputed in most writings on solid mechanics. As a reminder, we now provide a very brief statement of them.

Each of the terms of dissipation in $(18 a, b)$ is interpreted as a product of a generalized force" $x_{\alpha}$ and a generalized flux $J_{\alpha}$.

$$
\dot{\Theta}=J_{\alpha} x_{\alpha}
$$

Each $J_{\alpha}$ is the rate of change of an extensive quantity; it is a function of the local state e and of all forces:

$$
J_{\alpha}=F_{J}(u, a, \alpha ; x)
$$

By the nature of their construction, these relations must be homogeneous in the sense that $\mathrm{J}_{\alpha}=0$ for $\mathrm{X}=0$.

All relations of the type of equation (20a) constitute rate equations. They must be discovered by experiment and their knowledge, added to the appropriate set of conservation laws, and the fundamental equation, results in a closed system of partial differential equations. Their solution in the form

$$
u(x, t), a(x, t), \alpha(x, t),
$$

subject to prescribed boundary conditions, is the required irreversible process. The union of one fundamental equation of state and of the set of rate equations can be called the constitutive law of the system."

In many physical situations the relations (20a) are discovered to be linear:

Whe term generalized force used here applies to a different concept than that applied earlier to $F$.

\# This term is used to describe different concepts in other versions of thermodynamics. The present formalism creates a systematic basis for its discovery in actual problems. 


$$
J_{\alpha}=L_{\alpha \beta}(u, a, \alpha) \times X_{\beta} .
$$

When the relations are linear, the factors $L_{\alpha \beta}$ obey the Onsager-Casimir relations

with

$$
L_{\alpha \beta}=\varepsilon\left(X_{\alpha}\right) \varepsilon\left(X_{\beta}\right) L_{\alpha \beta}
$$

$$
\varepsilon\left(X_{\alpha}\right), \quad \varepsilon\left(X_{\beta}\right)=\left\{\begin{array}{l}
+1 \text { if } X_{\alpha} X_{\beta} \text { are even functions } \\
-1 \text { if } X_{\alpha} X_{\beta} \text { are odd functions }
\end{array}\right.
$$

of the microscopic velocities of particles. Hence the matrix $L_{\alpha \beta}$ may be symmetrical, antisymmetrical or mixed. In isotropic systems linear homogeneous relations can exist only between generalized forces of the some tensorial rank." In non-isotropic systems the proper symmetries must be incorporated.

Many authors, predominantly mechanicians of solids, vehemently deny the reality of the Onsager-Casimir relations. A very thorough review in the field of fluid mechanics conducted by D.G. Miller leaves very little doubt of their soundness when correctly applied.

Some authors maintain that in the local-state formalism the force-flux relations must be linear, a view not shared by this author. This is contradicted in at least one branch-chemical kinetics in which nonlinear relations predominate. Here, most probably, the authors take the phrase "local equilibrium" too literally or else, unjustifiably generalize certain conclusions valid in the analysis of the Boltzmann equation of kinetic theory.

- For some obscure reason this statemesit derivable from the general transformation properties of tensors is erroneously designated as Curie's principle. 


\subsection{A digression}

Some pedagogues may be interested in an alternative form of the ClausiusDuhem inequality, or, rather-Equation (18). We restrict its validity to isothermal situations and note that

$$
\int_{V} \rho T(d s / d t) d V=\dot{Q}^{\circ}
$$

is the rate at which heat is transferred in a reversible process. The second term multiplied by $\mathrm{T}=$ const can be transformed by the use of Gauss's theorem, to yield

$$
\int_{V}\left(\partial q_{k} / \partial x_{k}\right) d V=\oint_{\partial V} q_{k} v_{k} d A=\dot{Q} .
$$

Here $v_{k}$ is the outward normal, and $A$ the surface area. Paying attention to the sign convention in elementary thermodynamics, we find that the integral (24b) is equal to the heat rate in the physical space. Hence

$$
\dot{\mathrm{Q}}^{\circ}-\dot{\mathrm{Q}}=\mathrm{T} \dot{\Theta}
$$

In the primitive notation of elementary engineering thermodynamics, this is equivalent to

$$
d Q^{\circ} \cdot d Q=T d \Theta
$$

or, since

$$
d U=d Q^{\circ}-d W^{\circ}=d Q \cdot d W \text {, so that } d Q^{\circ} \cdot d Q=d W^{\circ} \cdot d W,
$$

we also have

$$
d W^{\circ}-d W=T d \Theta
$$

Equations $(26 a, b)$ have an interesting physical interpretation, restricted to isothermal processes, according to which the energy dissipation $\mathrm{T} \dot{\Theta}$ is equal to the "uncompensated" heat $\mathrm{dQ}^{\circ}$ - dQ or, what amounts to the same, also to the difference 
between the ideal ("reversible") and real work. Some people would call it "lost" or "dissipated" work.

\section{Nature of the approximation}

We now revert to the observation made in Section 4.1. in conjunction with Figure 4. The principle of local state assumes that

$$
\bar{T}=T \text { and } \bar{S}=S \text {, }
$$

whereas the attempt to visualize the transiton in the figure shows that

$$
S \cdot \bar{S}=\sigma>0 \text { and that } \bar{T} \cdot T=\theta \neq 0 .
$$

Thus, Equation (27) and its predecessor (15a) must be regarded as approximations whose adoption is justified by the long years of great success in the field of fluid mechanics where it rules supreme.

The numerical values of $\sigma$ and the difference $\theta$ cannot be calculated in terms of the present theory. For this a higher-order formulation is needed. Some people will find it in so-called extended irreversible thermodynamics where it can be shown that $\sigma$ and $\theta$ in (27a) are of higher order compared with the other terms in the equations.

The quantities $\sigma$ and $\theta$ represent the consequences of the dissipation due to the relaxation effects in the transition $n \rightarrow e$ in Figure 4. These can be measured by a relaxation time $\tau$ or, more precisely, by the longest of the several in existence, paying attention only to its order of magnitude. It is useful to compare $\tau$ with the intrinsic relaxation times of the rates of the process, defined as

$$
\tau_{a}=\left(a-a_{0}\right) / \dot{a} \text {. }
$$

It is also useful to express the relation of $\tau$ to $\tau_{\mathrm{a}}$ through the dimensionless Deborah number

$$
\mathrm{De}=\tau / \tau_{\mathrm{a}}
$$


The present author has proposed to use the Deborah number (28b) as a measure of the distance between the nonequilibrium state $n$ and its accompanying equilibrium state $e$. This may also be called the degree of nonequi'ibrium of $\mathrm{n}$. 


\section{Captions to Figures}

Figure 1. Physical space.

Figure 2. Reversible and irreversible creation of dislocations.

Volterra cut.

Figure 3. The Gibbsian phase (state) space.

Figure 4. Adiabatic transition from nonequilibrium (n) to accompanying equilibrium state (e).

Figure 5. Geometric illustration of the transition $n \rightarrow e$. 


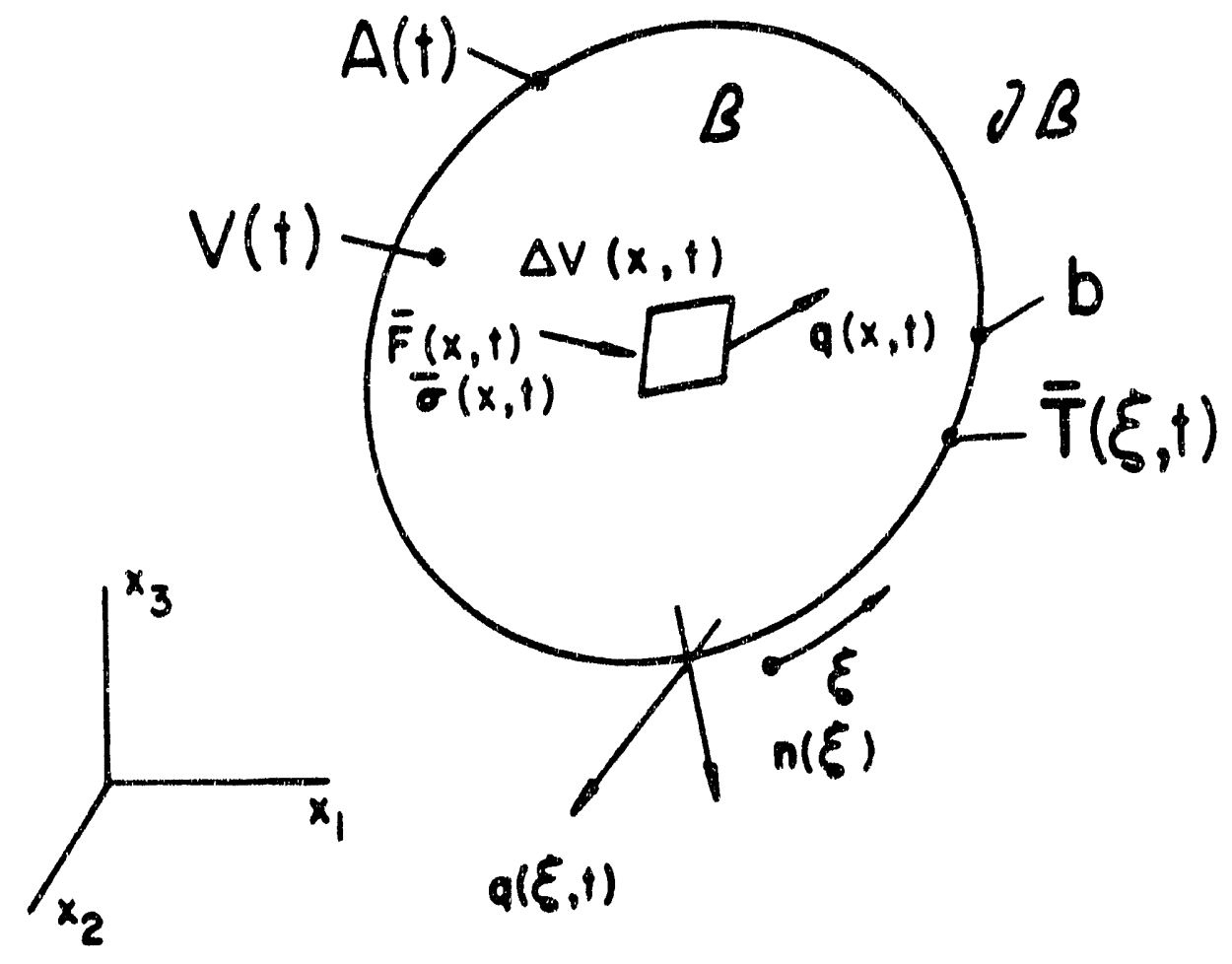

Figure 1. Physical space. 
irreversible
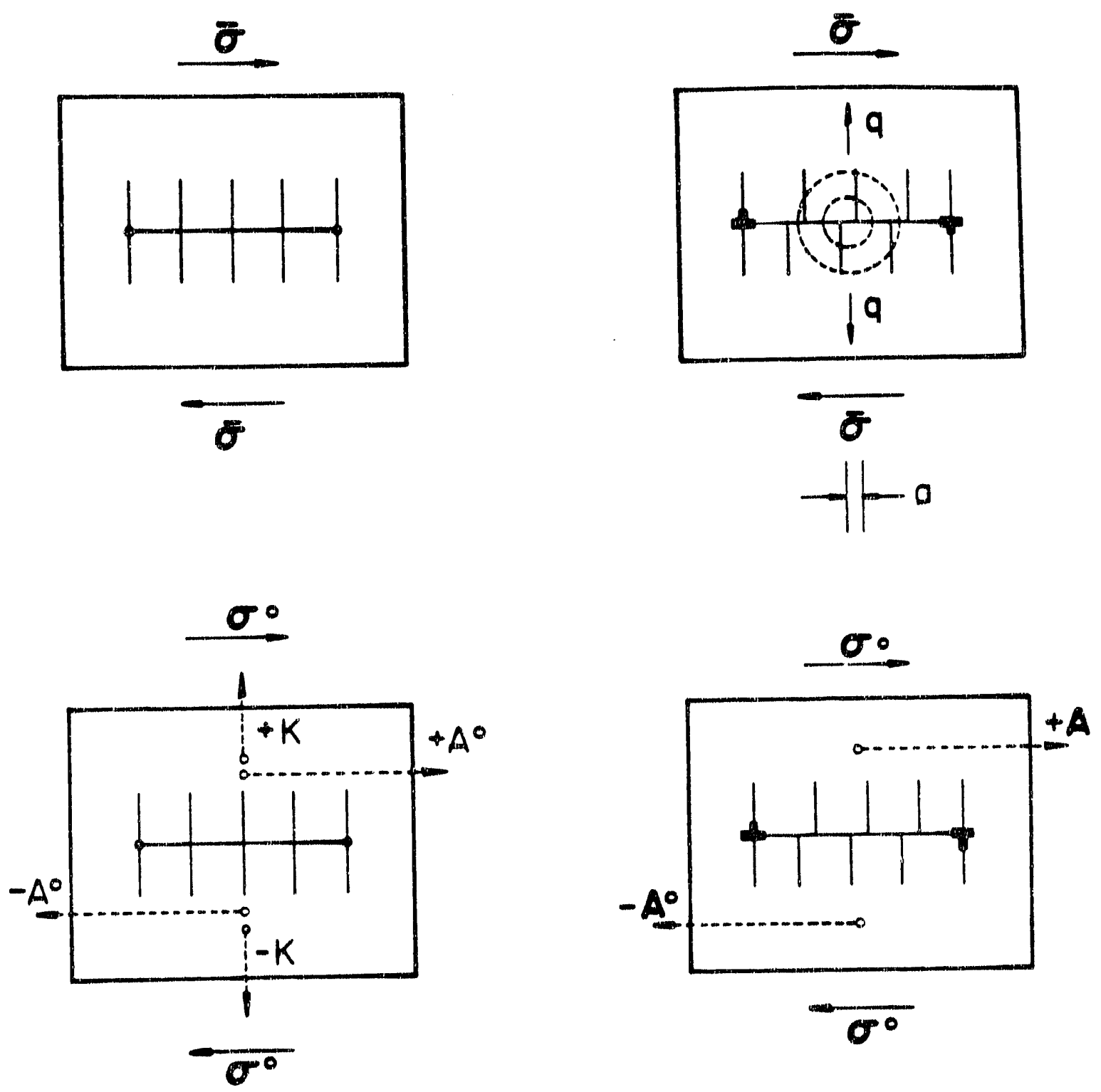

reversible

(Volterro cut)

Gedonkenexperiment

Figure 2. Reversible and irreversible creation of dislocations. Volterra cut. 


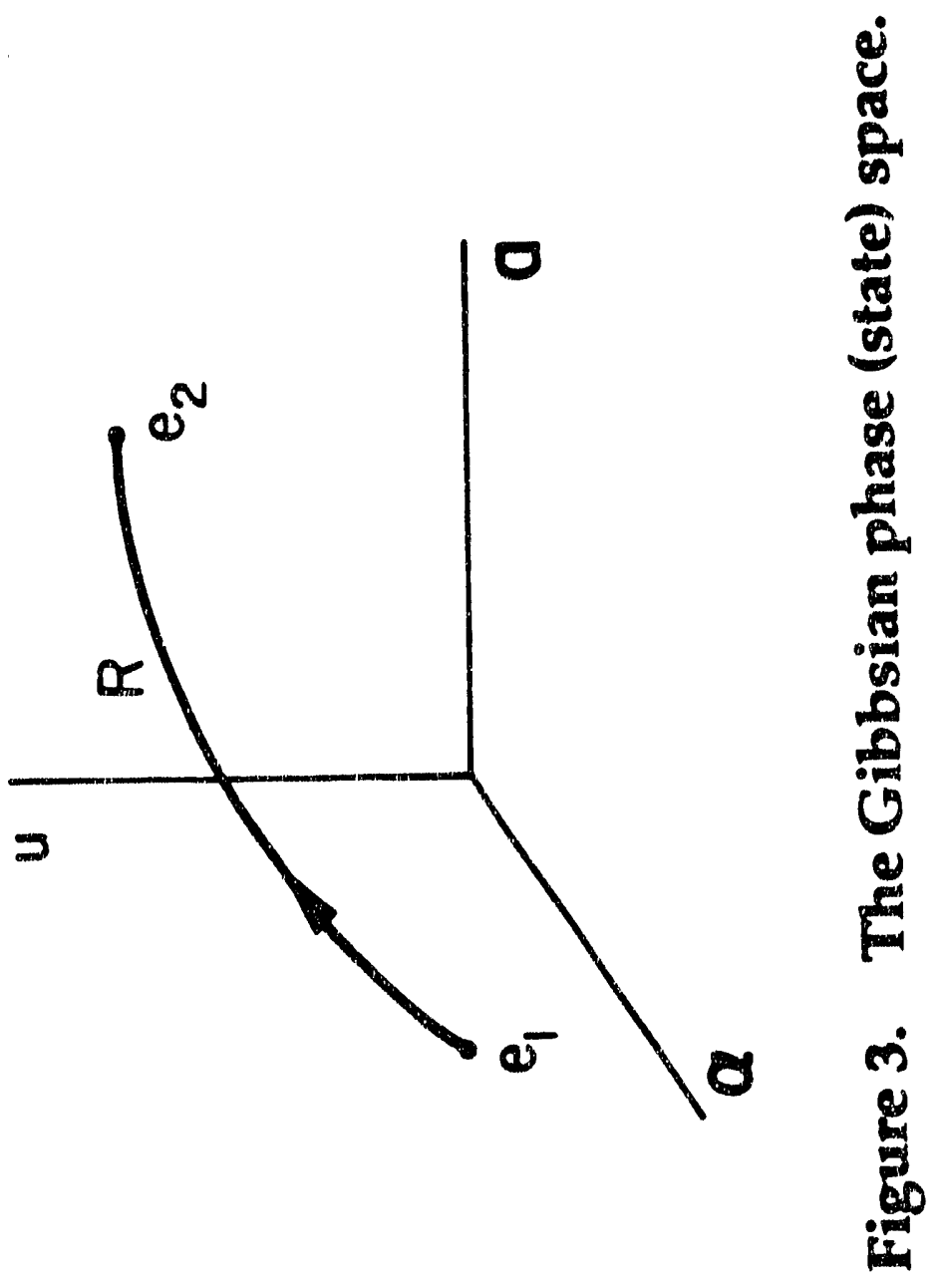




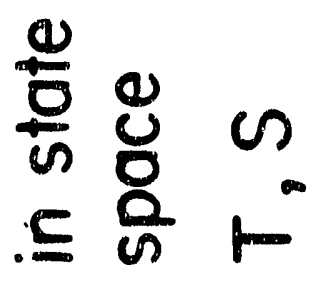

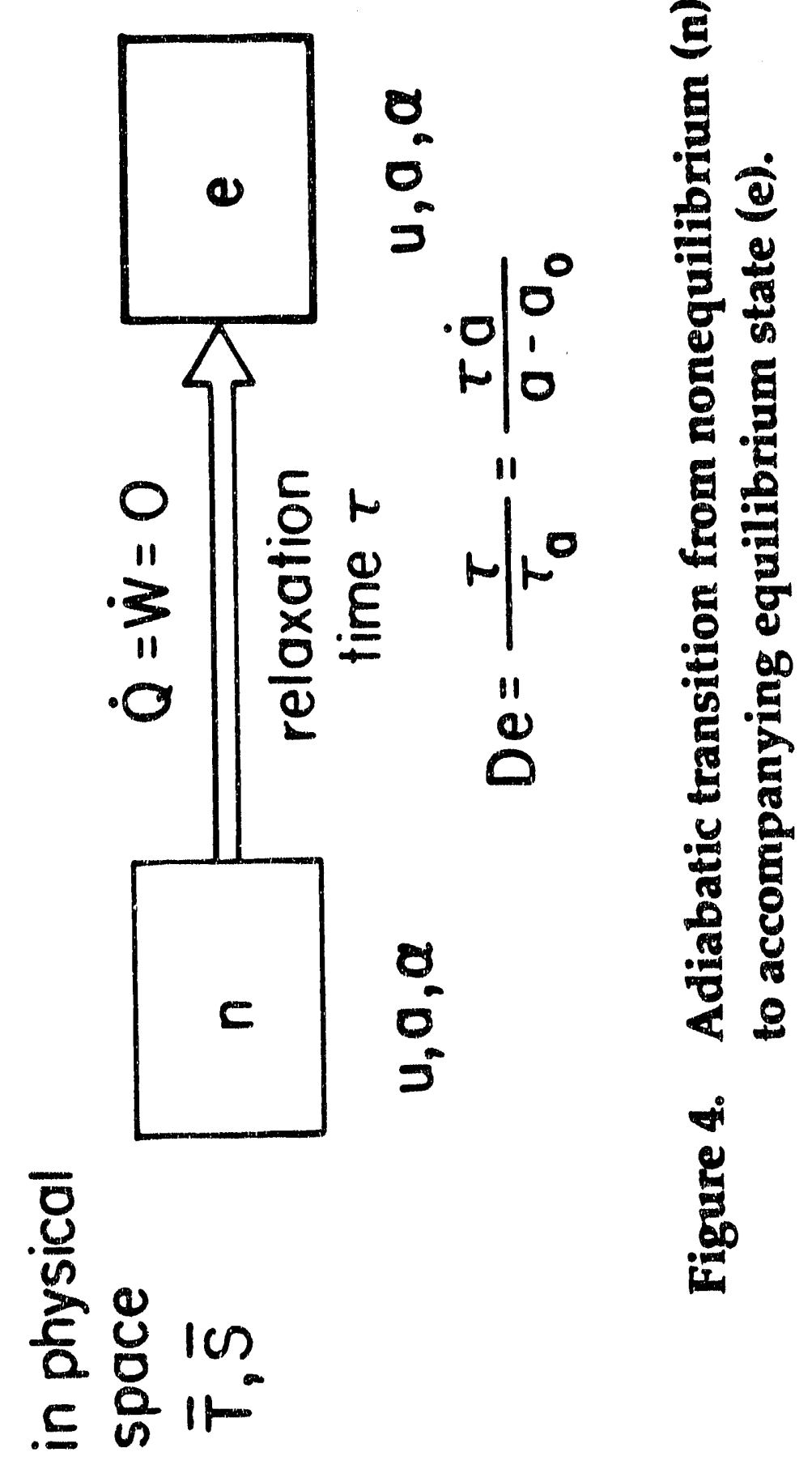




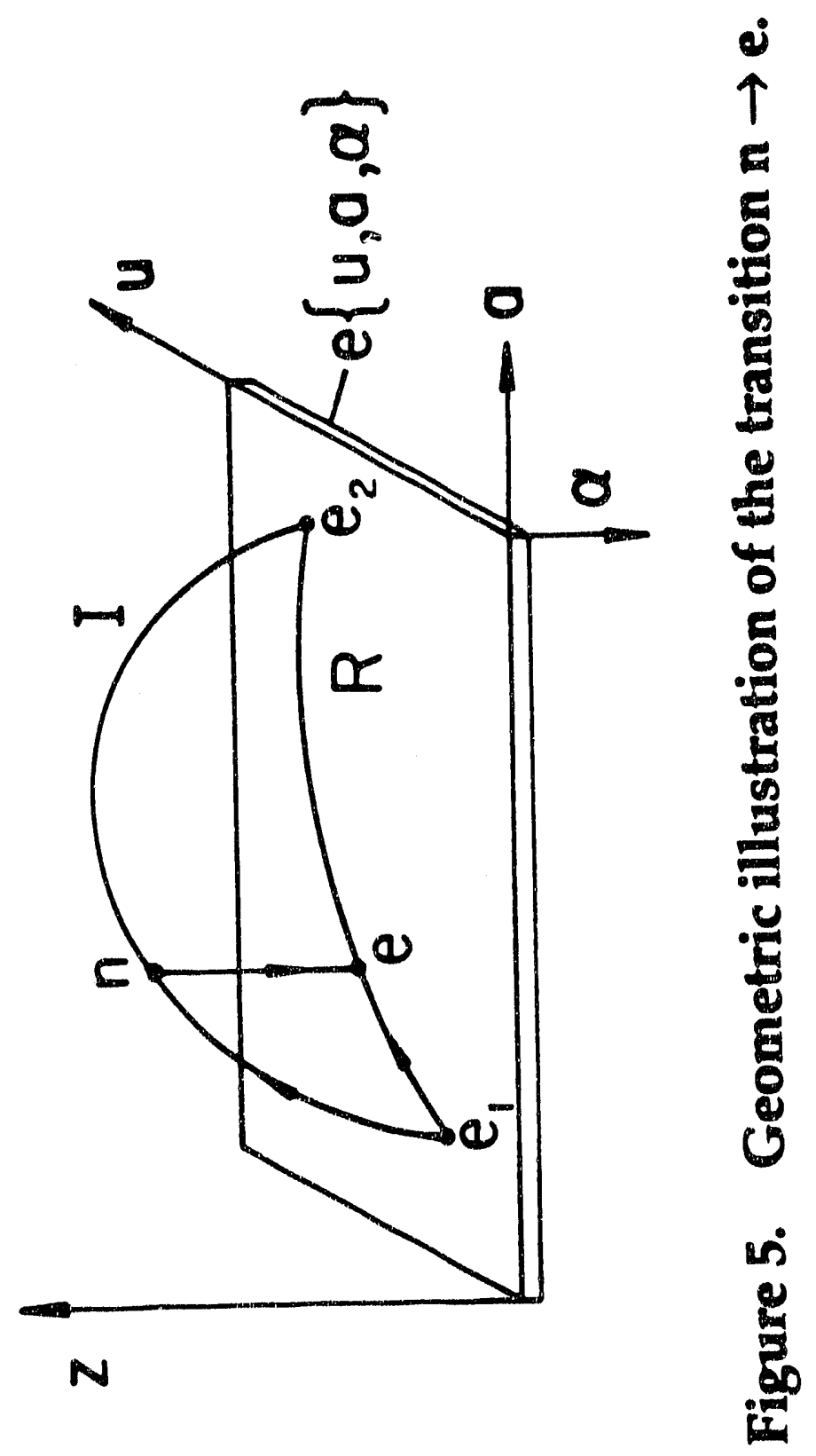


Appendix 1.

The reason why declaring entropy a "primitive quantity" without further discussion is not acceptable is best stated by quoting Arnold Sommerfeld:

Word definitions have very little meaning; physically significant definitions are obtained as soon as we prescribe a way of measuring the quantity in question. Such a prescription need not contain the details of practical procedure, but merely state a way to measure the quantity in principle."

Mechanics (transl. M. O. Stern)

Academic Press, 1952, p. 6. 


\section{Appendix 2.}

\section{P. W. Bridgman's insight}

I believe that in general the analysis of such systems will be furthered by the recognition of a new type of large-scale thermodynamic parameter of state, namely the parameter of state which can be measured but not controlled. ... These parameters are measurable, but they are not controllable, which means that they are coupled to no external force variable which might provide the means of control. And not being coupled to a force variable, they cannot take part in mechanical work.

The Nature of Thermodynamics

Harvard U. Press, 1941

and Harper \& Brothers, 1961 

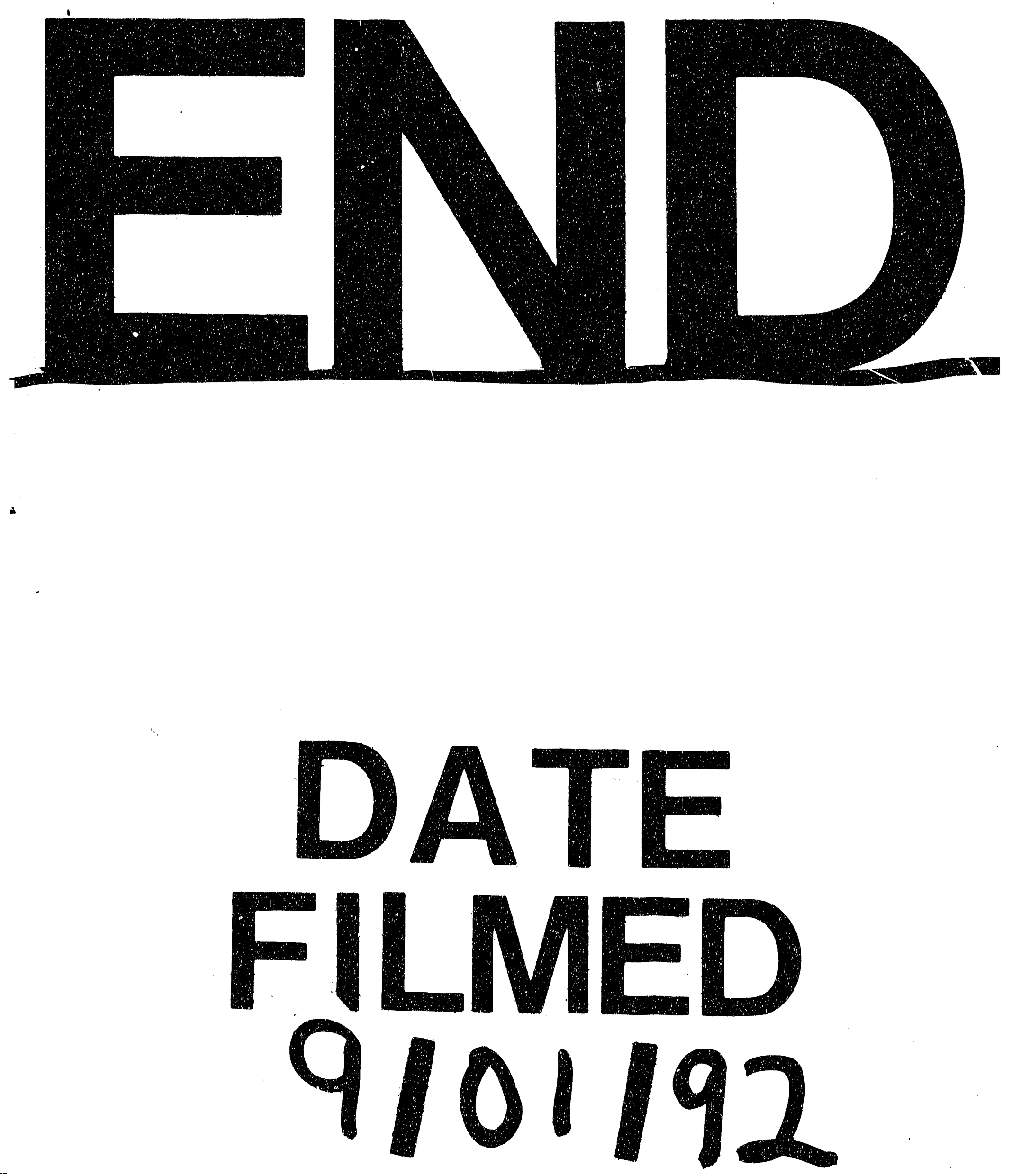
I 